\title{
Flexural Phonons in Free-Standing Graphene
}

\author{
Eros Mariani and Felix von Oppen \\ Institut für Theoretische Physik, Freie Universität Berlin, Arnimallee 14, 14195 Berlin, Germany
}

(Received 13 August 2007; published 20 February 2008)

\begin{abstract}
Rotation and reflection symmetries impose that out-of-plane (flexural) phonons of freestanding graphene membranes have a quadratic dispersion at long wavelength and can be excited by charge carriers in pairs only. As a result, we find that flexural phonons dominate the phonon contribution to the resistivity $\rho$ below a crossover temperature $T_{x}$ where we obtain an anomalous temperature dependence $\rho \propto T^{5 / 2} \ln T$. The logarithmic factor arises from renormalizations of the flexural-phonon dispersion due to coupling between bending and stretching degrees of freedom of the membrane.
\end{abstract}

DOI: 10.1103/PhysRevLett.100.076801

PACS numbers: 73.63.- b, 72.10.- d, 81.05.Uw

Introduction. - The experimental realization of monolayers of graphite, termed graphene, has opened new horizons in the physics of two-dimensional electron systems (2DES) $[1,2]$. Unlike conventional 2DES, the low-energy electronic band structure of graphene [3-5] is described by a massless Dirac equation with velocity $v$. The $4 \times 4$ matrix structure of the Dirac equation reflects the two sublattices of the graphene honeycomb lattice in combination with a valley degeneracy due to the presence of two Dirac cones within the Brillouin zone. Pioneering experiments on this novel two-dimensional electron system have shown that the Dirac nature of carriers induces an anomalous integer quantum Hall effect as well as a finite conductivity at vanishing carrier density $[1,2]$.

Recently, it has become possible to experiment on freestanding graphene sheets $[6,7]$ which provide a realization of a two-dimensional (2D) solid. Studies of the stability of 2D solids against thermal fluctuations date back to early works by Peierls and Landau $[8,9]$ who pointed out the absence of true long-range translational order. Much later, it was understood [10] that nevertheless, quasi-long-range translational order can persist up to a finite-temperature Kosterlitz-Thouless transition. While these works focus on in-plane lattice distortions, freestanding membranes also support out-of-plane ones. It is believed that there is a lowtemperature flat phase even in the presence of out-of-plane distortions [11], which gives way to a crumpled phase at high temperatures [12].

Within the low-temperature flat phase, in-plane, and outof-plane long-wavelength distortions can be described by the elastic Lagrangian density $[11,13]$,

$$
\mathcal{L}=\frac{\rho_{0}}{2}\left(\dot{\mathbf{u}}^{2}+\dot{h}^{2}\right)-\frac{1}{2} \kappa_{0}\left(\nabla^{2} h\right)^{2}-\mu u_{i j}^{2}-\frac{1}{2} \lambda u_{k k}^{2}
$$

in terms of the mass density $\rho_{0}$, the out-of-plane distortions $h(\mathbf{r})$, the in-plane ones $\mathbf{u}(\mathbf{r})$ and the strain tensor $u_{i j}=\frac{1}{2} \times$ $\left[\partial_{i} u_{j}+\partial_{j} u_{i}+\left(\partial_{i} h\right)\left(\partial_{j} h\right)\right]$. The elastic constants $\lambda$ and $\mu$ characterize the in-plane rigidity of the lattice, $\kappa_{0}$ the bending rigidity. Both the absence of a $(\nabla h)^{2}$ term in Eq. (1) and the appearance of the out-of-plane distortions $h(\mathbf{r})$ in the strain tensor are consequences of the rotational symmetry of the membrane in the space.

The elastic Lagrangian in Eq. (1) shows a distinct difference between in-plane and out-of-plane (flexural) phonons. Indeed, to quadratic order in the displacements $h(\mathbf{r})$ and $\mathbf{u}(\mathbf{r})$, both longitudinal and transverse in-plane phonons have a linear dispersion $\omega_{\mathbf{q}}^{(l)}=v^{(l)} q$ and $\omega_{\mathbf{q}}^{(t)}=v^{(t)} q$ with group velocities $v^{(l)}=\left[(2 \mu+\lambda) / \rho_{0}\right]^{1 / 2}$ and $v^{(t)}=$ $\left[\mu / \rho_{0}\right]^{1 / 2}$. In contrast, due to rotational symmetry, flexural phonons obey a quadratic dispersion $\omega_{\mathbf{q}}^{(h)}=\alpha q^{2}$ with $\alpha=$ $\left[\kappa_{0} / \rho_{0}\right]^{1 / 2}$. In-plane and flexural phonons also differ in their coupling to the charge carriers in graphene. While the coupling is conventional for in-plane phonons, the reflection symmetry $h \rightarrow-h$ demands that out-of-plane displacements enter only quadratically into the Dirac Hamiltonian. Consequently, charge carriers can excite flexural phonons only in pairs.

Because of these differences, we find that flexural modes dominate the phonon contribution to the resistivity of freestanding doped graphene membranes below a crossover temperature $T_{x}$. The transport scattering rate of Dirac fermions diverges logarithmically for a strictly quadratic dispersion of flexural modes. This divergence is cut off by the coupling between bending and stretching degrees of freedom captured by the Lagrangian in Eq. (1). At finite temperature, this coupling renormalizes the bending rigidity of the membrane, inducing a stiffening of the flexuralmode dispersion at long wavelengths. Including this physics within a simple one-loop RG, we find that the contribution of flexural phonons to the resistivity of graphene membranes scales as $T^{5 / 2} \ln T$.

Graphene.-The band structure of graphene is well approximated by the tight-binding Hamiltonian

$$
H=-t \sum_{\langle i j\rangle}\left[c_{i}^{\dagger} c_{j}+c_{j}^{\dagger} c_{i}\right]
$$

on a honeycomb lattice. Here, $t$ is the hopping matrix element, $c_{i}$ annihilates an electron on lattice site $i$, and only nearest-neighbor hopping has been included. The 2D hexagonal lattice consists of two identical sublattices $A$ and 
$B$, and thus two sites per unit cell. We denote the vectors connecting a $B$ site with the neighboring $A$ sites as $\mathbf{e}_{1}=$ $a(-1,0), \mathbf{e}_{2}=a(1 / 2, \sqrt{3} / 2)$, and $\mathbf{e}_{3}=a(1 / 2,-\sqrt{3} / 2)$ where $a$ is the bond length. The band structure of the Hamiltonian in Eq. (2) has zero energy (corresponding to the Fermi energy at half filling) at two inequivalent points in the Brillouin zone, which we choose to be at $\mathbf{k}_{ \pm}=$ $\pm \mathbf{k}_{D}$, with $\mathbf{k}_{D}=2 \pi /(3 \sqrt{3} a)(\sqrt{3}, 1)$. In the vicinity of these Dirac points, the spectrum is described by the $4 \times$ 4 Dirac Hamiltonian

$$
H=\hbar v \mathbf{\Sigma} \cdot \mathbf{k}
$$

with velocity $v=3 t a / 2$. The $2 \mathrm{D}$ wave number $\mathbf{k}$ is measured from the Dirac point. The Hamiltonian in Eq. (3) acts on four-component spinors $\left(u_{A, \mathbf{k}}^{+}, u_{B, \mathbf{k}}^{+}, u_{B, \mathbf{k}}^{-}, u_{A, \mathbf{k}}^{-}\right)$of Bloch amplitudes in the space spanned by the sublattices $(A / B)$ and Dirac points $(+/-)$. The matrices $\Sigma_{x, y}=\Pi_{z} \otimes \sigma_{x, y}$ denote components of a vector $\boldsymbol{\Sigma}$. $\left(\Pi_{i}\right.$ and $\sigma_{j}$ are Pauli matrices acting in the spaces of the Dirac points and the sublattices, respectively.) It is also useful to introduce a corresponding vector $\Lambda$ by $\Lambda_{x, y}=\Pi_{x, y} \otimes \sigma_{z}$ and $\Lambda_{z}=$ $\Pi_{z} \otimes \sigma_{0}[14]$.

Electron-phonon coupling. - The dominant electronphonon coupling arises from distortion-induced modifications of the bond lengths and hence the hopping amplitude [15-18]. The electron-phonon coupling can then be described in terms of a fictitious gauge field $\mathbf{A}(\mathbf{r})$ entering into the Dirac Hamiltonian, $H=v \mathbf{\Sigma} \cdot(\mathbf{p}+e \mathbf{A}(\mathbf{r}, t))$, where $\mathbf{p}$ denotes the momentum. In terms of the strain tensor $u_{i j}$, the gauge field $\mathbf{A}(\mathbf{r})$ takes the form [19]

$$
e \mathbf{A}(\mathbf{r}, t)=\Pi_{z} \otimes \mathbf{1} \frac{\hbar}{t} \frac{\partial t}{\partial a}\left[\begin{array}{c}
u_{x y} \\
\frac{1}{2}\left(u_{x x}-u_{y y}\right)
\end{array}\right] .
$$

The combination of components of the strain tensor entering into $\mathbf{A}(\mathbf{r})$ reflects the symmetry of the underlying honeycomb lattice. The factor of $\Pi_{z}$ implies that the associated fictitious magnetic field points in opposite directions at the two Dirac points, as required by timereversal symmetry. From the definition of the strain tensor, we conclude that electrons couple linearly to the in-plane phonons and quadratically to flexural ones. Figure 1 shows the corresponding electron-phonon vertices.
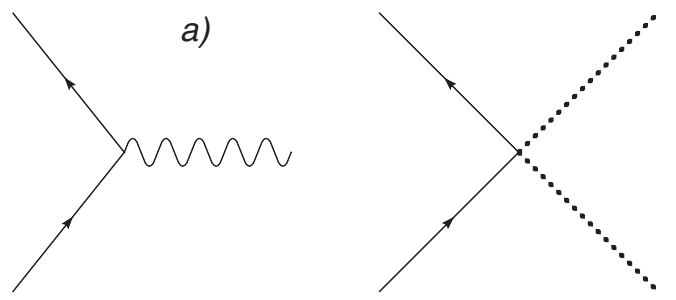

b)

FIG. 1. Electron-phonon vertices. (a) Coupling of electrons to in-plane phonons. (b) Coupling of electrons to flexural phonons. Straight lines correspond to electrons, wavy (dashed) lines to inplane (flexural) phonons.
At sufficiently low temperatures, only long-wavelength phonons contribute to the resistivity and scattering between different Dirac cones can be neglected. Thus, we can restrict attention to the vicinity of, say, the Dirac point $\mathbf{k}_{+}$where the Hamiltonian reduces to a $2 \times 2$ Hamiltonian in the $A-B$ sublattice space.

The electron-phonon coupling $H_{\text {ep }}$ corresponding to Eq. (4) can be expressed in second quantization formalism after expanding the in-plane and out-of-plane distortions into Fourier series as $\mathbf{u}(\mathbf{r})=\sum_{\mathbf{q}} \mathbf{u}_{\mathbf{q}} e^{i \mathbf{q} \cdot \mathbf{r}}$ and $h(\mathbf{r})=$ $\sum_{\mathbf{q}} h_{\mathbf{q}} e^{i \mathbf{q} \cdot \mathbf{r}}$, decomposing $\mathbf{u}_{\mathbf{q}}$ into longitudinal and transverse components,

$$
\mathbf{u}_{\mathbf{q}}=u_{\mathbf{q}}^{(l)} \hat{\mathbf{q}}+u_{\mathbf{q}}^{(t)} \hat{\mathbf{z}} \times \hat{\mathbf{q}},
$$

and quantizing the amplitude of the distortions as

$$
u_{\mathbf{q}}^{(\nu)}=\sqrt{\frac{\hbar}{2 M \omega_{\mathbf{q}}^{(\nu)}}}\left(a_{\mathbf{q}}^{(\nu)}+a_{-\mathbf{q}}^{(\nu) \dagger}\right) .
$$

Here $M$ is the atomic mass and $a_{\mathbf{q}}^{(\nu)}$ the annihilator of a phonon of type $\nu(\nu=l, t, h)$ with wave number $\mathbf{q}$. Thus

$$
\begin{aligned}
H_{\mathrm{ep}}= & \sum_{\mathbf{k}, \mathbf{k}^{\prime}, \mathbf{q}} \sum_{\nu=l, t} V_{\mathbf{q}}^{(\nu)} c_{B, \mathbf{k}}^{\dagger} c_{A, \mathbf{k}^{\prime}}\left(a_{\mathbf{q}}^{(\nu)}+a_{-\mathbf{q}}^{(\nu) \dagger}\right) \delta_{\mathbf{k}^{\prime}, \mathbf{k}-\mathbf{q}} \\
& +\sum_{\mathbf{k}, \mathbf{k}^{\prime}, \mathbf{q}, \mathbf{q}^{\prime}} V_{\mathbf{q}, \mathbf{q}^{\prime}}^{(h)} c_{B, \mathbf{k}}^{\dagger} c_{A, \mathbf{k}^{\prime}}\left(a_{\mathbf{q}}^{(h)}+a_{-\mathbf{q}}^{(h) \dagger}\right) \\
& \times\left(a_{\mathbf{q}^{\prime}}^{(h)}+a_{-\mathbf{q}^{\prime}}^{(h) \dagger}\right) \delta_{\mathbf{k}^{\prime}, \mathbf{k}-\mathbf{k}-\mathbf{q}^{\prime} \mathbf{q}^{\prime}}+\text { H.c. }
\end{aligned}
$$

in terms of the coupling terms

$$
\begin{aligned}
V_{\mathbf{q}}^{(l)} & =-\epsilon q e^{-2 i \phi} \sqrt{\frac{\hbar}{2 M \omega_{\mathbf{q}}^{(l)}}}, \\
V_{\mathbf{q}}^{(t)} & =-i \epsilon q e^{-2 i \phi} \sqrt{\frac{\hbar}{2 M \omega_{\mathbf{q}}^{(t)}}}, \\
V_{\mathbf{q}, \mathbf{q}^{\prime}}^{(h)} & =-\frac{1}{2} i \epsilon q q^{\prime} e^{-i\left(\phi+\phi^{\prime}\right)} \frac{\hbar}{2 M \sqrt{\omega_{\mathbf{q}}^{(h)} \omega_{\mathbf{q}^{\prime}}^{(h)}}},
\end{aligned}
$$

where $\epsilon=(3 a / 4) \partial t / \partial a, c_{B, \mathbf{k}}$ is the annihilator of an electron in a Bloch state of sublattice $B$ and wave-vector $\mathbf{k}$, and $\phi\left(\phi^{\prime}\right)$ the angle of $\mathbf{q}\left(\mathbf{q}^{\prime}\right)$ with respect to the $x$ axis.

Thus we reach an interesting competition: In-plane phonons are strongly coupled to electrons (the vertex is first order in the phonons) but their linear dispersion yields a linearly vanishing density of states at small energy. In contrast, flexural phonons are weakly coupled to electrons (their vertex is second order in the phonons) but their dispersion is quadratic with constant density of states. In the following, we quantitatively analyze the consequences of this competition for the temperature dependence of the resistivity. The latter is determined by

$$
\rho=\frac{2}{e^{2} v^{2} \nu_{F}} \frac{1}{\tau_{\mathrm{tr}}}
$$


( $\nu_{F}$ is the electronic density of states at the Fermi level) in terms of the transport scattering rate

$$
\frac{1}{\tau_{\mathrm{tr}}}=\frac{2 \pi}{\hbar} \sum_{f}\left|M_{f i}\right|^{2}(1-\cos \theta) \delta\left(E_{f}-E_{i} \pm \hbar \omega\right)
$$

of Dirac fermions due to absorption (or emission) of phonons. Here, $\theta$ is the scattering angle of the fermions, $|i\rangle$ and $|f\rangle$ the initial and final scattering states, $E_{i}$ and $E_{f}$ the initial and final electronic energies, $\hbar \omega$ the energy of the absorbed (emitted) phonons, and $M_{f i}=\left\langle f\left|H_{\mathrm{ep}}\right| i\right\rangle$.

We consider the doped regime $E_{F} \tau \gg 1$ (here $1 / \tau$ denotes the total scattering rate due to disorder and phonon scattering), where electronic transport can be described quasiclassically and employ the conventional quasielastic approximation (valid when $E_{F} \gg k_{B} T$ ) of neglecting the phononic contribution to energy conservation, as the typical phonon energies are small compared to $E_{F}$. Moreover, we focus on sufficiently low temperatures so that we can restrict attention to the quadratic (linear) region of the dispersion of flexural (in-plane) phonons.

The phonon contribution to the resistivity is typically a small correction to a dominant disorder contribution. Nevertheless, its temperature dependence makes the phonon-induced resistivity experimentally discernible [20] from the $T$-independent disorder one [21].

Flexural phonons. - Scattering of Dirac fermions by flexural phonons requires absorption (or emission) of two phonons, say with wave numbers $\mathbf{q}$ and $\mathbf{q}^{\prime}$. Thus, the corresponding initial and final scattering states are $|i\rangle=|\mathbf{k}, \sigma\rangle \otimes\left|n_{\mathbf{q}}^{(h)}\right\rangle \quad$ and $\quad|f\rangle=\left|\mathbf{k}+\mathbf{q}+\mathbf{q}^{\prime}, \sigma^{\prime}\right\rangle \otimes$ $\left|n_{\mathbf{q}}^{(h)}-1, n_{\mathbf{q}^{\prime}}^{(h)}-1\right\rangle, \quad$ where $\quad n_{\mathbf{q}}^{(h)}=\left[\exp \left(\hbar \omega_{\mathbf{q}}^{(h)} / k_{B} T\right)-\right.$ $1]^{-1}$ denotes the Bose distribution function of the flexural phonons and $|\mathbf{k}, \sigma\rangle=1 / \sqrt{2}\left(\sigma e^{-i \xi} c_{A, \mathbf{k}}^{\dagger}+c_{B, \mathbf{k}}^{\dagger}\right) \mid$ vac $\rangle$ is a Dirac fermion state with momentum $\mathbf{k}$ and chirality $\sigma$. (Here, $\xi$ is the angle between $\mathbf{k}$ and the $x$ axis and $|\mathrm{vac}\rangle$ is the electronic vacuum). Thus, $\left|M_{f i}\right|^{2}=\left|V_{\mathbf{q}, \mathbf{q}^{\prime}}^{(h)}\right|^{2}[1-$ $\left.\sigma \sigma^{\prime} \cos \left(\xi+\xi^{\prime}+2 \phi+2 \phi^{\prime}\right)\right] / 2$. The summation over final states requires integration over $\mathbf{q}$ and $\mathbf{q}^{\prime}$, while averaging over the direction of the incoming electron will suppress the oscillatory term in $\left|M_{f i}\right|^{2}$. Using $a_{\mathbf{q}}^{(\nu)}\left|n_{\mathbf{q}}^{(\nu)}\right\rangle=$ $\left(n_{\mathbf{q}}^{(\nu)}\right)^{1 / 2}\left|n_{\mathbf{q}}^{(\nu)}-1\right\rangle, \quad E_{i}=\hbar v k=E_{F}, \quad$ and $\quad E_{f}=\hbar v \mid \mathbf{k}+$ $\mathbf{q}+\mathbf{q}^{\prime} \mid$, we obtain

$$
\frac{1}{\tau_{\mathrm{tr}}^{(h)}}=\sum_{\mathbf{q}, \mathbf{q}^{\prime}} \frac{\pi\left|V_{\mathbf{q}, \mathbf{q}^{\prime}}^{(h)}\right|^{2}}{\hbar}(1-\cos \theta) n_{q}^{(h)} n_{q^{\prime}}^{(h)} \delta\left(\Phi_{\mathbf{q}+\mathbf{q}^{\prime}}\right),
$$

where $\quad \Phi_{\mathbf{q}+\mathbf{q}^{\prime}}=\hbar v\left|\mathbf{k}+\mathbf{q}+\mathbf{q}^{\prime}\right|-E_{F} \quad$ and $\quad\left|V_{\mathbf{q}, \mathbf{q}^{\prime}}^{(h)}\right|^{2}=$ $\hbar^{2} \epsilon^{2} /\left(16 M^{2} \alpha^{2}\right)$ is independent of wave numbers due to the quadratic dispersion of flexural phonons. The rate in Eq. (11) is formally singular at small $q, q^{\prime}$ since both Bose distributions diverge as $T / q^{2}$. Rescaling momenta by $\sqrt{T}$ and introducing a cutoff $q_{c}$ at small wave numbers (to be specified below), we obtain the scattering rate

$$
\frac{1}{\tau_{\mathrm{tr}}^{(h)}} \simeq \frac{C^{(h)}}{32 \pi^{2}} \frac{\hbar \omega_{k_{F}}^{(h)}}{\kappa_{0}}\left(\frac{k_{B} T}{\hbar \omega_{k_{F}}^{(h)}}\right)^{5 / 2} \ln \left(\frac{k_{B} T}{\hbar \omega_{q_{c}}^{(h)}}\right),
$$

where $C^{(\nu)}=\epsilon^{2} \hbar k_{F}^{4} /\left(4 \rho_{0} E_{F} \hbar \omega_{k_{F}}^{(\nu)}\right)$. The unusual $T^{5 / 2}$ scaling implies that scattering from flexural phonons dominates the phonon contribution to the resistivity at low temperatures. Indeed, the conventional phonon contribution to the resistivity due to in-plane phonons scales as $T^{4}$, which is the direct two-dimensional analog of the $T^{5}$ law in bulk solids.

Equation (12) shows that the scattering rate from flexural phonons diverges logarithmically for a strictly quadratic phonon dispersion. For clean elastic membranes, a low-momentum cutoff arises from the coupling terms between bending and stretching degrees of freedom of the membrane which are included in the Lagrangian equation (1). These lead to long-wavelength corrections to the elastic constants and hence the phonon dispersions [11]. Indeed, it is these renormalizations that are responsible for the stability of the flat phase at low temperatures.

In order to study the renormalization of the bending rigidity by the coupling of bending and stretching modes, we integrate out the in-plane distortions in Eq. (1) and obtain an effective energy functional for the flexural modes alone [11],

$$
F=\frac{1}{2} \int d \mathbf{r}\left[\kappa_{0}\left(\nabla^{2} h\right)^{2}+\frac{K_{0}}{4}\left[P_{\alpha \beta}^{\perp}\left(\partial_{\alpha} h\right)\left(\partial_{\beta} h\right)\right]^{2}\right],
$$

where $\quad K_{0}=4 \mu(\mu+\lambda) /(2 \mu+\lambda) \quad$ and $\quad P_{\alpha \beta}^{\perp}=$ $\left(\epsilon_{\alpha l} \epsilon_{\beta k} \partial_{l} \partial_{k}\right) / \nabla^{2}$ is a transverse projector. This effective energy functional contains a four-leg interaction between flexural distortions. Treating this quartic term to one-loop order (depicted in Fig. 2) in a conventional momentumshell RG, one obtains the flow equation

$$
\frac{d \kappa}{d q}=-\frac{3}{16 \pi} \frac{K_{0} k_{B} T}{\kappa q^{3}},
$$

with $q$ denoting the running shell wave vector.

The flow equation Eq. (14) is readily solved, and the resulting scaling of $\kappa$ with $q$ is

$$
\kappa(q)=\kappa_{0} \sqrt{1+q_{c}^{2} / q^{2}}
$$

in terms of the temperature-dependent momentum scale $q_{c}=\left[3 K_{0} k_{B} T /\left(8 \pi \kappa_{0}^{2}\right)\right]^{1 / 2}$. Thus, the flexural-phonon dispersion is quadratic for $q \gg q_{c}$. In contrast, for $q \ll q_{c}$, thermal fluctuations effectively stiffen the membrane and,

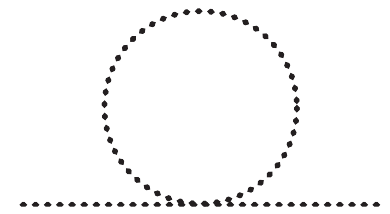

FIG. 2. One-loop correction to the bending rigidity due to the effective interaction between flexural modes. 
within the simple one-loop analysis presented here, we find a renormalized dispersion $\omega_{q \ll q_{c}}^{(h)}=\left(\kappa_{0} q_{c}\right)^{1 / 2} q^{3 / 2}$. It is this renormalization of the flexural-phonon dispersion at long wavelength which removes the singularity in the phonon scattering rate Eq. (12). We therefore identify the low-momentum cutoff $q_{c}$ entering into Eq. (12) with this momentum scale.

As a result, we find that the temperature cancels from the argument of the logarithm in Eq. (12). This analysis is approximate in that it is restricted to one-loop order and that it neglects the flow of the elastic constants for stretching deformations. While the stiffening against bending deformation would survive inclusion in a more refined treatment, the precise power-law dependence of $q_{c}$ on temperature would change. As a result, the argument of the logarithm would become temperature dependent, resulting in an overall $T^{5 / 2} \ln T$ scaling of the flexural-phonon contribution to the resistivity.

Crossover temperature. - In order to estimate the crossover temperature $T_{x}$ below which phonon scattering of Dirac fermions is dominated by flexural modes, we note that an analogous calculation yields

$$
\frac{1}{\tau_{\mathrm{tr}}^{(\nu)}} \simeq C^{(\nu)}\left(\frac{k_{B} T}{\hbar \omega_{k_{F}}^{(\nu)}}\right)^{4}
$$

for the transport scattering rate from in-plane phonons $(\nu=l, t)$. Thus, the crossover temperature obtained from a comparison of Eqs. (12) and (16) becomes

$$
T_{x}=\frac{1}{k_{B}}\left(\frac{\ln \left(k_{B} T / \hbar \omega_{q_{c}}^{(h)}\right)}{8(2 \pi)^{2}} \frac{\hbar \omega_{k_{F}}^{(t) 5}}{\kappa_{0}\left(\hbar \omega_{k_{F}}^{(h)}\right)^{5 / 2}}\right)^{2 / 3},
$$

independent of the Fermi energy (i.e., the doping level) within the considered doped regime. With typical parameters for graphene [23], we obtain $T_{x} \simeq 70 \mathrm{~K}$, yielding a significant temperature range over which our predictions for the resistivity can be experimentally tested.

Conclusions. - For clean graphene membranes, longwavelength renormalizations of their elastic properties due to thermal fluctuations are crucial in order to obtain a finite transport scattering rate and hence resistivity contribution $\rho \propto T^{5 / 2} \ln T$ from scattering by flexural phonons. In the presence of disorder, the elastic properties of the membrane are renormalized even at zero temperature $[24,25]$. Indeed, the recent experimental observations of rippling [6] suggest that disorder, inside or close to the 2D membrane, exists in present graphene membranes. Such disorder-induced renormalizations of the elastic moduli may compete with the renormalizations by thermal fluctuations and lead to a temperature-independent saturation of the cutoff $q_{c}$ at low temperatures. A detailed study of the effects of disorder in this context remains an important topic for future research.

We are grateful to F. Guinea, T. Nattermann, L. Peliti, and Ady Stern for instructive discussions. This work was supported in part by DIP. F. v. O. enjoyed the hospitality of the KITP Santa Barbara (supported in part by NSF Grant No. PHY99-07949) and E. M. acknowledges the hospitality of the Weizmann Institute of Science (supported by Grant No. RITA-CT-2003-506095).

[1] K.S. Novoselov et al., Science 306, 666 (2004); K.S. Novoselov et al., Nature (London) 438, 197 (2005).

[2] Y. Zhang et al., Nature (London) 438, 201 (2005); Y. Zhang et al., Phys. Rev. Lett. 94, 176803 (2005).

[3] P. R. Wallace, Phys. Rev. 71, 622 (1947).

[4] J. Gonzales, F. Guinea, and M. A. H. Vozmediano, Nucl. Phys. B406, 771 (1993).

[5] R. Saito, G. Dresselhaus, and M. S. Dresselhaus, Physical Properties of Carbon Nanotubes (Imperial College Press, London, 1998).

[6] J. C. Meyer et al., Nature (London) 446, 60 (2007).

[7] J. Scott Bunch et al., Science 315, 490 (2007).

[8] R. E. Peierls, Ann. Inst. Henri Poincaré 5, 177 (1935).

[9] L. D. Landau, Phys. Z. Sowjetunion 11, 26 (1937).

[10] V. L. Berezinskii, Sov. Phys. JETP 32, 493 (1971); J. M. Kosterlitz and D. J. Thouless, J. Phys. C 6, 1181 (1973).

[11] D. R. Nelson and L. Peliti, J. Phys. (Paris) 48, 1085 (1987).

[12] M. Paczuski, M. Kardar, and D. R. Nelson, Phys. Rev. Lett. 60, 2638 (1988).

[13] L. D. Landau and E. M. Lifshitz, Theory of Elasticity (Pergamon, New York, 1986).

[14] E. McCann et al., Phys. Rev. Lett. 97, 146805 (2006).

[15] L. M. Woods and G. D. Mahan, Phys. Rev. B 61, 10651 (2000).

[16] J. L. Manes, Phys. Rev. B 76, 045430 (2007).

[17] A.H. Castro Neto and E.A. Kim, arXiv:cond-mat/ 0702562.

[18] For the effects of in-plane phonons on the graphene band structure, see M. Calandra and F. Mauri, Phys. Rev. B 76, 205411 (2007); C. H. Park et al., Phys. Rev. Lett. 99, 086804 (2007); W.-K. Tse and S. Das Sarma, Phys. Rev. Lett. 99, 236802 (2007).

[19] See also A. K. Geim and M. I. Katsnelson, Phil. Trans. R. Soc. A 366, 195 (2008).

[20] For a recent preliminary measurement using substratebased graphene, see Y.-W. Tan et al., Eur. J. Phys. Special Topics 148, 15 (2007).

[21] Other temperature-dependent contributions may arise from weak localization and Altshuler-Aronov corrections. However, both of these require a significant density of atomic scale defects [22] which is inconsistent with the observed doping dependence of the resistivity in highmobility graphene. The latter favors a predominance of Coulomb scatterers, instead. Weak localization is also easily eliminated by weak magnetic fields.

[22] V. V. Cheianov and V. I. Fal'ko, Phys. Rev. Lett. 97, 226801 (2006).

[23] Typical parameters for graphene sheets are $\kappa_{0}=1 \mathrm{eV}$, $\mu \simeq 4 \lambda=9 \mathrm{eV} \AA^{-2}, \hbar \alpha=35 \mathrm{meV} \AA^{2}$.

[24] L. Radzihovsky and D. R. Nelson, Phys. Rev. A 44, 3525 (1991).

[25] D. C. Morse, T. C. Lubensky, and G. S. Grest, Phys. Rev. A 45, R2151 (1992); D. C. Morse and T. C. Lubensky, Phys. Rev. A 46, 1751 (1992). 